Boss at the Dudley Observatory on August 7, and August 12, 1880. According to the elements given above, a maximum occurred on Aug. 12, 1880. As shown on about thirty photographs, the change is slow at the time of maximum, and the star probably remains bright for at least two days.

19030I. This star has been observed on 506 photographs, taken between May 22, 3888 and November 9, 1906. The provisional elements for times of maximum, J. D. $2411873.865+13^{d} .75 E$, have been derived from a preliminary study of these observations. The spectrum appears to change. At maximum, it is of Class $G$, and the distribution of the light is even from the bands $H$ and $K$ to the line $H \beta$. At or near minimum, the spectrum resembles Class $\mathrm{K}$, and the portion of the spectrum from $H \varepsilon$ to $H \gamma$ bas become much fainter than the portion from $H \gamma$ to $H \beta$. It thus appears as if the variation may be due to a diminution in the rays of shorter wave length.

230258. Observations of this star have been made on 24 photographs, laken between August 8, 1899 and December 19, 1906. The period appears to be about five days. At maximum, this star is as bright as $+57^{\circ} 2694$, magnitude 9.2. At minimum, it becomes at least one magnitude fainter.

Harvard College Observatory, Cambridge, Mass., I907 May Io.

Edward C. Pickering.

\title{
Notiz betreffend die Sekularstörungen von (433) Eros.
}

In den Nr. 4178.79 der Astr. Nachr., in dem Artikel: - Secular pertubations of Erose außerte Herr C. J. Merfield die Meinung, da $B$ ein paar Fehler in meinen Rechnungen (Sekulare Marsstörungen in der Bewegung des Eros, Krakau, I g06) vorhanden seien. Ich untersuchte diese Stellen, und es zeigte sich, da $b$ in dem Ausdrucke fur $\left[\frac{\mathrm{d} L}{\mathrm{~d} t}\right]_{00}$ ein Glied falsch genommen ist, deswegen anderte sich dieser Ausdruck fur alle vier Planeten.

Es wird nämlich:

$$
\begin{aligned}
& \text { für Mars }\left[\frac{\mathrm{d} L}{\mathrm{~d} t}\right]_{\infty 0}=+0.27514 \\
& \text { far Erde , }=+7.44828 \\
& \text { far Jupiter } \quad=-17.29578 \\
& \text { für Saturn, , }=-0.7455^{6}
\end{aligned}
$$

Diese Resultate stimmen ziemlich nahe mit den Resultaten von Herrn Merfield uberein.

Dann kommt noch ein Fehler in der Tafel II in dem Ausdrucke $\log W_{0}$ fur $0^{\circ}$. Es soll dort heißen: $9.07707 \mathrm{n}$, was mit dem entsprechenden Ausdrucke in den Tafeln des Herrn Merfield fast ubereinstimmt. Deswegen muß man auch fur $0^{\circ}$ die entsprechenden Glieder in $W_{0} \cos u$ und $W_{0} \sin u$ ändern, nämlich in +0.11932 und -0.00490 . Daraus folgt, daß die Sekularstörungen der Erde andere Werte erhalten: $\frac{\mathrm{d} i}{\mathrm{~d} t}=-0.00085, \frac{\mathrm{d} \Omega}{\mathrm{d} t}=-3.22923, \frac{\mathrm{d} \pi}{\mathrm{d} t}=+2.62889$ was aber nicht stark von den fruher angegebenen abweicht.

Da der Hauptfehler die Ausdrucke für die Länge betrifft, so bleiben die Untersuchungen der kritischen Epochen noch ziemlich tichtig, und es muß nur die Zeit der größten Annäherung in der kritischen Epoche als falsch angesehen werden.

Krakau, Sternwarte, 1907 Juni 7.

W. Dzienulski.

Beobachtungen der Kometen $1906 \mathrm{~d}$ (Finlay) und $1906 \mathrm{~g}$.

\begin{tabular}{l|l|l|l|l|l|l|l|l|l}
\hline I906 & M. Z. Wien & $\Delta \alpha$ & $\Delta \delta$ & Vgl. & $\alpha$ app. & $\log p . \Delta$ & $\delta$ app. & $\log p . \Delta \mid$ & Red. ad l.app. \\
\hline
\end{tabular}

Finlayscher Komet $1906 \mathrm{~d}$.

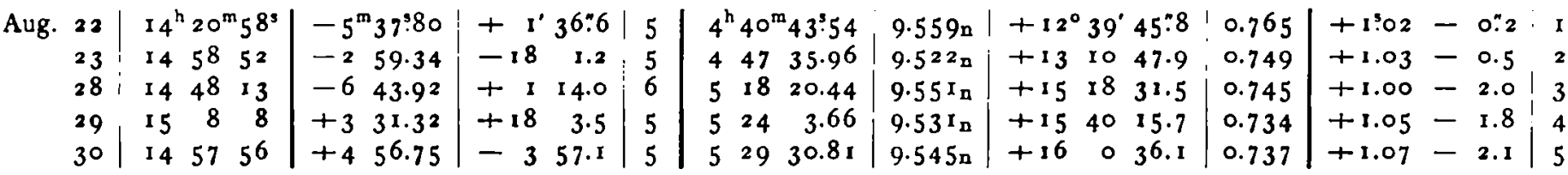

Komet $1906 \mathrm{~g}$

Nov. 20| Iy 43 II $|-235.99|+3$ I 1.0| $5|10438.39| 8.663_{n}|+252426.6| 0.531|+1.75-17.6| 6$

Mittlere Örter der Vergleichsterne für 1906.0.

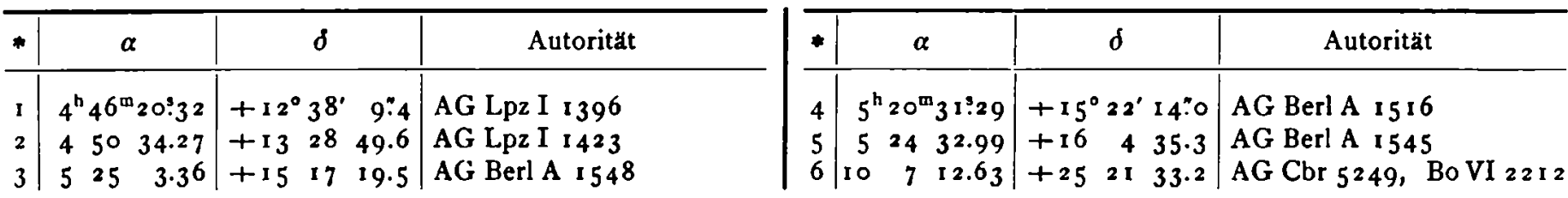

Jeder dieser zwei Kometen erschien im 6-zoll. Refraktor als ein ziemlich großer runder Nebel mit einer helleren Mitte, in welcher beim ersten Kometen eine kernartige Verdichtung, wenn auch nicht immer deutlich, zu sehen war, wăhrend eine solche beim zweiten nur in seltenen Momenten und auch da nicht mit Sicherheit erkannt werden konnte. Der zweite Komet war überdies mehr blaß und an den Răndern sehr unsicher begrenat. 